\title{
Socio-cognitive training impacts emotional and perceptual self- salience but not self-other distinction
}

\author{
RUNNING HEAD: EMOTIONAL \& PERCEPTUAL SALIENCE
}

Henryk Bukowski ${ }^{1,2 *}$ (hbbukowski@gmail.com),

Boryana Todorova ${ }^{1}$ (btodorovaaa@gmail.com),

Magdalena Boch ${ }^{1,3}$ (magdalena.boch@univie.ac.at),

Giorgia Silani ${ }^{4}$ (giorgia.silani@univie.ac.at),

\& Claus Lamm¹ (claus.lamm@univie.ac.at)

${ }^{1}$ Social, Cognitive and Affective Neuroscience Unit, Department of Cognition, Emotion, and Methods, University of Vienna, Vienna, Austria

${ }^{2}$ Institute of Psychological Sciences, University of Louvain, Louvain-La-Neuve, Belgium

${ }^{3}$ Department of Cognitive Biology, Faculty of Life Sciences, University of Vienna, Vienna, Austria

${ }^{4}$ Department of Clinical and Health Psychology, University of Vienna, Vienna, Austria

*Corresponding authors:

Henryk Bukowski (permanent address)

Square de la Liberté, 10

7090 Braine-le-Comte

Belgium

Keywords: empathy, self-other distinction, imitation, self-salience, personal distress

The authors declare they have no competing interests. 


\section{EMOTIONAL \& PERCEPTUAL SALIENCE}

\section{Highlights:}

- Effect of a socio-cognitive training on self-other distinction and self-salience was tested

- Ss trained to imitate, inhibit imitation, or inhibit control stimuli in 2 experiments

- Unlike the original study, training did not influence self-other distinction

- Imitation-inhibition training increased self-salience in empathy and shape matching 


\title{
EMOTIONAL \& PERCEPTUAL SALIENCE
}

\begin{abstract}
:
It has been shown that 40 min of training on inhibition of imitative tendencies can successfully improve perspective-taking performance, suggesting an enhancement of self-other distinction transferrable from the motor to the cognitive domain (Santiesteban et al. 2012). This study examined whether and how this socio-cognitive training would improve self-other distinction in the emotional domain while controlling for effects on emotional and perceptual self-salience (i.e., the prioritized processing of the self-related stimuli). Across two experiments, participants were trained to either imitate, inhibit imitation, inhibit control stimuli, or were imitated. On the following day they completed an affective touch paradigm measuring both self-other distinction and emotional self-salience and a shape matching paradigm measuring perceptual self-salience. Results indicate no significant and consistent impact of training on self-other distinction performance but highlight an increased emotional and perceptual self-salience following training to inhibit imitative tendencies. These large sample-based results put into question whether the socio-cognitive training improves performance via enhanced self-other distinction and invites to consider self-salience as a complementary angle to explain the past, present, and future self-other distinction findings.
\end{abstract}




\section{EMOTIONAL \& PERCEPTUAL SALIENCE}

\section{Introduction}

Our actions and thoughts are so deeply influenced by other people's actions and thoughts that being able to control these influences is considered a key socio-cognitive ability, now often referred to as self-other distinction. Other people's thoughts and feelings have been demonstrated to influence our own thinking and feelings in perspective-taking and empathy paradigms, this influence is known as altercentric (or allocentric) bias, intrusion, or interference (Samson et al., 2010; Silani, Lamm, Ruff, \& Singer, 2013). Similarly, the importance of self-other distinction is demonstrated, where self-thoughts (e.g., a belief or knowledge) or self-feelings influence our inferences of what others think or feel by making them biased, erroneous, or slower to perform; which is typically referred to as egocentric bias, intrusion, or interference. Pioneering work demonstrating the difficulty in inhibit others' influences devised the so-called automatic imitation paradigm in order to measure individuals' susceptibility to imitative tendencies interfering with task performance (Brass, Bekkering, \& Prinz, 2001; Brass, Bekkering, Wohlschla, \& Prinz, 2000). Santiesteban et al. (2012) by showing that individuals trained to inhibit their imitative tendencies have a reduction of imitative interferences and an enhancement of perspective-taking performance, fed the hypothesis that a single unitary mechanism may be involved across different sociocognitive domains such as automatic imitation, empathy, and perspective-taking paradigms. The present study aimed to test whether the impact of this training extends to empathic performance while controlling for self-salience in the affective and perceptual domains.

Self-salience, also named self-prioritization, self-advantage, self-centeredness, or egocentrism, refers to the processing prioritization of self-related information over information pertaining to other people. Self-salience is well-known in the memory domain as the self-reference effect where information processed with explicit reference to the self are better remembered (Cunningham, Turk, Macdonald, Macrae, \& Neil Macrae, 2008; Rogers, Kuiper, \& Kirker, 1977; Sui, Humphreys, \& He, 2012). In contrast, self-salience in the perspective-taking domain is evidenced by the extent of egocentric bias (i.e., extent of interference by the self $=$ extent of selfprioritization) but the egocentric bias is also used as an index of self-other distinction (i.e., extent of interference by the self = extent of self-other distinction difficulties); which shows that selfsalience and self-other distinction are confounded in perspective-taking paradigms (Bukowski, 2014). The same logic applies to automatic imitation as it is unclear whether an observed strong interference results from poor self-other distinction or a low self-salience (since per extension it 


\section{EMOTIONAL \& PERCEPTUAL SALIENCE}

indicates a high salience of information related to the other person). Consequently, it remains unclear whether the impact of inhibition-imitation training stems from altered self-salience or altered self-other distinction. The only study to date investigating the positive effects of inhibitionimitation training on empathy (de Guzman, Bird, Banissy, \& Catmur, 2015) used two empathy measures devoid of task-demands to enforce self-other distinction: the first was corticospinal activity when observing physical pain inflicted to another person's and the second was score on a self-report questionnaire of trait empathy. Hence, this study undertook to answer this open question by examining the impact of the same training procedure on self-other distinction performance in empathy while controlling for self-salience. To this aim, self-other distinction in empathy was measured with the affective touch paradigm (Silani et al., 2013) by assessing the extent emotional states induced via visuotactile stimulations onto the self and another person provoke biases when rating how another or oneself feels (cf. Materials for a detailed description of the paradigm). Moreover, self-salience was measured within the same paradigm by assessing the extent selfexperienced emotions are rated as more intense than the emotions experienced by the other person (Bukowski, Silani, Riva, Tomova, \& Lamm, 2016). Finally, an additional, more widely validated, measure of self-salience was used: The shape-matching task assess the extent out of three pairings between a shape (triangle, square, or circle) and a label (you, your best friend, an unfamiliar person) the self-shape pairing has the highest performance at verifying whether the presented pairing is correct (cf. Materials for full description).

The present study consisted of two experiments with an identical procedure (except for experiment 1 that had an additional and novel training condition) in order to reliably examine the impact of the socio-cognitive training on self-other distinction and self-salience. In short, participants were first trained, and after $24 \mathrm{~h}$ (as in the original study) they completed the affective touch and the shape matching task. In addition, dispositional empathy was assessed on day 1 and mood state was assessed right before starting the affective touch task.

Experiments 1 and 2 were conducted at the University of Vienna, at the Faculty of Psychology and complied with local ethical regulations and the Declaration of Helsinki (2013, latest revision). The raw and processed data can be found online on https://osf.io/pcv3u/. None of the methods, procedures, and analyses were pre-registered. The methods and results of two experiments are presented separately and followed by a results section where datasets from the two experiments are collapsed. 


\section{EMOTIONAL \& PERCEPTUAL SALIENCE}

\section{Experiment 1}

\subsection{Material and methods}

\subsubsection{Sample:}

Only female participants were recruited for consistency with previous work (Riva, Triscoli, Lamm, Carnaghi, \& Silani, 2016; Silani et al., 2013) and to increase statistical homogeneity, given sex/gender differences on this task had been documented as well (Tomova et al., 2014). We ran a power analysis using the training effect size $\left(\eta_{\mathrm{p}}{ }^{2}=.12\right)$ from the only published study that used the socio-cognitive training before the conduction of the present study (Santiesteban et al., 2012). The analysis conducted with $G^{*}$ Power $3.1(\alpha=0.05,1-\beta=0.95,3$ groups, 2 repeated measurements) indicated a requirement of at least 11 participants per group. We decided to double the required sample size and recruited 99 healthy adults, resulting in a final sample of 91 participants $\left(M_{\text {age }}=\right.$ 21.58; $\left.S D_{\text {age }}=3.71\right)$ after exclusion of 8 participants due to dropouts or data loss caused by technical problems. The sample was randomly distributed between 4 training groups: the imitation $(N=24)$, imitation-inhibition $(N=21)$, and control-inhibition $(N=22)$ as in Santiesteban et al. (2012) and a fourth novel training "be-imitated" group $(N=24)$ inspired from a study showing impact of participants' finger movements being imitated on empathy (De Coster, Andres, \& Brass, 2014). Inclusion criteria were an absence of past or current psychiatric diagnosis. Participants received a course credit with the psychology bachelor curriculum in return of their participations.

\subsubsection{Procedure:}

All participants came to the laboratory twice on two consecutive days and were tested in pairs. They were contacted prior to their first session on day 1 to provide them with the general information about the study, to ensure they fit inclusion criteria and to schedule an appointment paired with another participant. Both participants followed the same procedure. Upon arrival on day 1, participants received further information about the procedure, their related rights and provided their informed consents. Then they were split in two separate but adjacent rooms. The participants completed one of the four socio-cognitive training procedures followed by two selfreport questionnaires (Interpersonal Reactivity Index, IRI; the cognitive empathy subscale of the Empathy Quotient, cEQ). On the next day (day 2, 24 hours after the training) participants again received information about the tasks to follow and provided their informed consents before entering the separated test rooms. Then they completed: (1) the training for the affective touch task, (2) 


\section{EMOTIONAL \& PERCEPTUAL SALIENCE}

Positive and Negative Affect Scale (PANAS), (3) the affective touch task, and (4) the shape matching task. At last participants were debriefed separately.

\subsubsection{Materials:}

Socio-cognitive training: The socio-cognitive (SC) training was devised by Santiesteban et al. (2012) by adapting the 'automatic imitation' task, a stimulus-response matching task measuring how task-irrelevant finger lifting of another person interferes with participants' own finger lifting in response to numeric cues (Brass et al., 2001). Specifically, the instructions of the original automatic imitation task were changed to form: (1) a "imitation" group where participants had to imitate the other person's finger lifting, (2) a "imitation-inhibition" group where participants had to lift the opposite finger as the other person's finger lifting (i.e., the participant's index finger when the other person's major was being lifted, and vice-versa), (3) a "imitation-control" group where participants had to lift the key of opposite colour that the colour of a sticker superimposed on the static hand (e.g., they had to lift a finger from the red key when a green sticker appears on screen), and (4) a "be-imitated" group where participants were instructed to lift either their index or major finger and to observe their finger lifting being imitated by the other person's identical finger. The last condition is a novel addition to the original SC training and was motivated by the finding of enhanced empathic response to another person's pain when being imitated by another person with the same automatic imitation materials (De Coster et al., 2014). For all 4 training condition participants completed 6 blocks of 72 trials, which lasted about $40 \mathrm{~min}$.

Affective touch task: The affective touch task (also known as the visuo-tactile empathy task or emotional egocentricity task), developed by Silani et al. (2013), measures participants' ratings of the pleasantness or unpleasantness of visuotactile stimulations that two paired participants receive simultaneously. Participants received distinct tactile stimulations and were informed via a screen about what each participant was being touched by. Right after the stimulation, they were instructed to rate either the other participant's emotional state or their own emotional state on a scale ranging from unpleasant to pleasant. Critically, in half of the trials both participants experienced similarly valenced emotional state (congruent; both pleasant or unpleasant stimulations) where in the other half they experienced an opposite emotional state (incongruent; one received a pleasant, the other an unpleasant stimulation). The rated target and the similarity of emotional states formed two orthogonal factors: the target perspective to take (self vs. other) and 


\section{EMOTIONAL \& PERCEPTUAL SALIENCE}

the congruency between the two participants' emotional experiences (congruent vs. incongruent). Congruent and incongruent trials are mixed within two blocks, one block during which participants are instructed to systematically rate only the other person's emotional state and one block during which participants rate only their own emotional state. Each block consisted of 40 trials and each trial had the following sequence: (1) A fixation cross displayed on screen (range: 1800-5850 ms); (2) three seconds of pleasant (e.g., a rose or a feather) or unpleasant (e.g., a worm or a slug) tactile stimulations alongside with two pictures (400 x 400 pixels) depicting each participant's touch stimulus and a headline "You" or "Your colleague" above the respective pictures; (3) a two-second time window to rate the intensity and valence of the target person's emotional state via a finger touch on the screen over a visual analogue scale where the top-end was a manikin face expressing pleasantness and the bottom-end was a face expressing unpleasantness. Participants were informed that the materials they were touched with were innocuous and inert but were instructed to imagine the emotional state as if the target was touched by the depicted stimulus. The order of the trials was pseudorandomized to avoid more than two consecutive trials of the same type and blocks order was counter-balanced across subjects. Before starting the task, participants were familiarized with the task via a practice block of 30 trials (10 pleasant, 10 unpleasant, 10 neutral) in which they received and rated visuotactile stimuli solely from the self-perspective (there was no picture of the other person's stimulus). This block allowed the participants to get a first-hand experience with all visuotactile stimulations before having to imagine how it would feel for another person. The whole task lasted about 25 min (Silani et al., 2013 for further details on the task).

The main effect of congruency captures self-other distinction performance by measuring the extent the ratings of the target person's emotional state are drawn towards the irrelevant person's emotional state when their emotional states are incongruent, which is quantified as less intense (i.e., closer to zero) ratings in the incongruent condition than the congruent condition. The ratings difference (i.e., median ${ }^{1}$ rating in congruent condition minus median rating incongruent condition) was therefore the index of self-other distinction performance

The main effect of perspective captures the relative salience of the self-experienced emotions over the other person's emotional experiences by measuring the extent the ratings were further away from zero (more pleasant or unpleasant) when rating the self-experienced emotion

\footnotetext{
${ }^{1}$ Medians were used instead of means (as in previous own work) because the majority of the participants in both experiments have produced at least one rating opposite to the expected valence (e.g., rating as unpleasant a trial that should be rated as pleasant), which excessively influences the mean but not the median.
} 


\section{EMOTIONAL \& PERCEPTUAL SALIENCE}

than when rating the other person's emotion. The rating difference (i.e., median rating in selfexperienced emotion condition minus median rating in the other person's emotion condition) was therefore the index of emotional self-salience.

At last, an interaction between congruency and perspective can be decomposed either in terms of egocentric and altercentric biases (or interference) when measuring self-other distinction performance separately for other person's perspective and self-perspective conditions, respectively, or in terms of perspective salience expressed either separately for congruent and incongruent trials.

Shape matching task: The shape matching task is a perceptual matching task devised by Sui, Humphreys and He (2012) measuring the extent participants are better (faster and more accurate) at verifying whether a shape (triangle, square, or circle) corresponds to the label "you", „your best friend“, or „unfamiliar person“ which has been learned via instructions and during a 12 trials practice block. Following a 500-ms fixation cross, one of the three shapes was presented along with one of the three labels for $100 \mathrm{~ms}$ following which participants had 800 to $1200 \mathrm{~ms}$ to press either of two keys to indicate whether the presented shape and label matched or mismatched. A 500-ms feedback was then presented (,Correct“, "Incorrect“, “Too late!“). Performed average accuracy was displayed at the end of each of the three blocks of 60 trials. Shape and label matched in half of the trials in the self, friend, and stranger conditions. Shape-label pairings were randomly determined at the beginning of the task. As in the original task, mean RT, rate of correct responses, and their combination as inverse efficiency scores (IES; i.e., RT/accuracy rate) were analyzed only for matching trials (no effect was found on the mismatching trials) to examine performance difference between the label-shape parings. Perceptual salience of the self (self-salience) is calculated by the extent of being more efficient at verifying the shape associated with the self than at verifying the other two pairings (friend and stranger). The task lasted about $20 \mathrm{~min}$.

PANAS: The Positive and Negative Affect Scale (Watson, Clark \& Tellegen, 1988) is a self-report mood scale with 10 items assessing positive emotions and 10 items assessing negative emotions ranging from 1 "Not at all or a very little" to 5 "Extremely". Separate scores are computed by summing the ratings of the positive and negative items, respectively, and by subtracting the two scores (positive - negative). Participants' mood was tested before beginning of the affective touch task but after the practice trials. 


\section{EMOTIONAL \& PERCEPTUAL SALIENCE}

IRI: The Interpersonal Reactivity Index (IRI) is a self-report questionnaire assessing four dimensions of empathy across 4 subscales of 7 items ranging from ranging from 1 "Does not describe me at all" to 5 "Describes me very well". The Perspective-Taking (PT) subscale refers to everyday tendencies to consider the perspective of other people. The Fantasy (FS) subscale assesses the individual's tendencies to identify themselves with fictional characters in movies, books, etc. The Empathic Concern (EC) subscale measures the tendencies to feel concern and compassion for others. The Personal Distress (PD) subscale measures the tendencies to feel anxious or discomfort while observing the distress of others.

Cognitive Empathy Quotient: The empathy quotient is a 60-item self-report instrument used for measuring empathy in adults (Baron-Cohen, \& Wheelwright, 2004) that consists of 40 empathy-related items and 20 distractor filler items. The items are scored on a 4-point scale ranging between strongly agree and strongly disagree. Lawrence, Shaw, Baker, Baron-Cohen, and David (2004) identified three factors: cognitive empathy, emotional reactivity and social skills. In this experiment only the cognitive empathy subscale (cEQ) comprising 11 items from EQ was used.

\subsection{Results}

\subsubsection{Group differences}

Group differences in age and scores of the IRI, cEQ, and PANAS were inspected across the 4 priming groups by performing one-way ANOVAs with the training group membership as between-subject factor. Age data of 9 participants was missing whereas questionnaires data of 3 participants (4 for PANAS) was missing. The ANOVA did not reveal any significant difference between training groups for age $(F(3,82)=1.274, p=.289)$, IRI perspective-taking $(F(3,88)=$ $0.406, p=.749)$, IRI fantasy $(F(3,88)=0.632, p=.596)$, IRI empathic concern $(F(3,88)=1.386$, $p=.253)$, IRI personal distress $(F(3,85)=0.340, p=.799)$, IRI global score $(F(3,88)=0.948, p=$ $.421), \operatorname{cEQ}(F(3,88)=0.481, p=.697)$, PANAS positive affect $(F(3,87)=0.335, p=.800)$, PANAS negative affect $(F(3,87)=1.060, p=.371)$ and PANAS global score $(F(3,87)=1.422, p=.242)$.

\subsubsection{Empathic performance}

Rates of erroneous valence ratings $(M=.051, S D=0.095)$ of two participants were at chance level (50\% chance getting the right valence) and were thus excluded from analyses.

The repeated-measures ANOVA of the ratings revealed a significant main effect of congruency $F(1,84)=14.490, p<.001, \eta_{p}{ }^{2}=.147$, with a lower median emotional intensity in the 


\section{EMOTIONAL \& PERCEPTUAL SALIENCE}

incongruent emotions condition, a non-significant main effect of perspective $F(1,84)=1.008, p=$ $.318, \eta_{p}{ }^{2}=.012$, a non-significant congruency by perspective interaction, $F(1,84)=1.271, p=$ $.263, \eta_{p}^{2}=.015$, with an egocentric bias numerically but not significantly higher than the altercentric bias $(p=.150)$. These findings replicate previous studies (Bukowski et al., 2019; Silani et al., 2013).

Of particular interests were the interactions of socio-cognitive training types with the aforementioned effects. We found a non-significant congruency by training interaction, $F(3,84)=$ $0.803, p=.496, \eta_{p}^{2}=.028$, indicating no difference in the extent of the self-other distinction bias across training groups, a marginally significant perspective by training interaction, $F(1,84)=2.387$, $p=.075, \eta_{p}{ }^{2}=.079$, indicating a trend for a difference in the extent of the relative emotional selfsalience across training groups, and a non-significant congruency by perspective by training interaction, $F(3,84)=0.542, p=.655, \eta_{p}^{2}=.019$. At last, there was no significant main effect of training groups over empathic performance, $F(3,84)=0.542, p=.655, \eta_{p}^{2}=.019$.

In order to further characterize the trend, we examined the perspective $\mathrm{x}$ training interaction by comparing the emotional self-salience across training groups with a series of two-sided $t$-tests for independent samples. The imitation-inhibition group had self-salience $(M=0.319, S D=1.152)$ significantly higher than the be-imitated group $(M=-0.598, S D=1.205, p=.036)$, marginally significantly higher than the inhibition-control group $(M=-0.557, S D=1.177, p=.062)$, and not significantly different from the imitation group $(M=0.199, S D=2.062, p=.776)$. The be-imitated group had a marginally significantly lower self-salience than the imitation group $(p=.070)$ and the inhibition-control and be-imitated groups were not significant $(p=.931)$. While these results indicate a numerically highest self-salience in imitation-inhibition, inspection of upper panel of Figure 1, which plots median ratings across all experimental conditions, suggests that the selfsalience difference is more pronounced on congruent trials. An ANOVA on self-salience for congruent trials only confirmed a significant difference across training groups, $F(3,84)=2.775, p$ $=.046$. Post-hoc analyses revealed a self-salience significantly higher in the imitation-inhibition group $(M=0.295, S D=1.103)$ than in the inhibition-control $(M=-0.707, S D=1.290, p=.015)$ and be-imitated groups $(M=-0.587, S D=1.142, p=.022)$ but no significant difference from the imitation group $(M=-0.128, S D=1.340, p=.254)$. 
Overall, we found no significant impact of training on self-other distinction performance. Instead, we found a high emotional self-salience in the counter-imitation group, especially when focusing on congruent emotions trials.
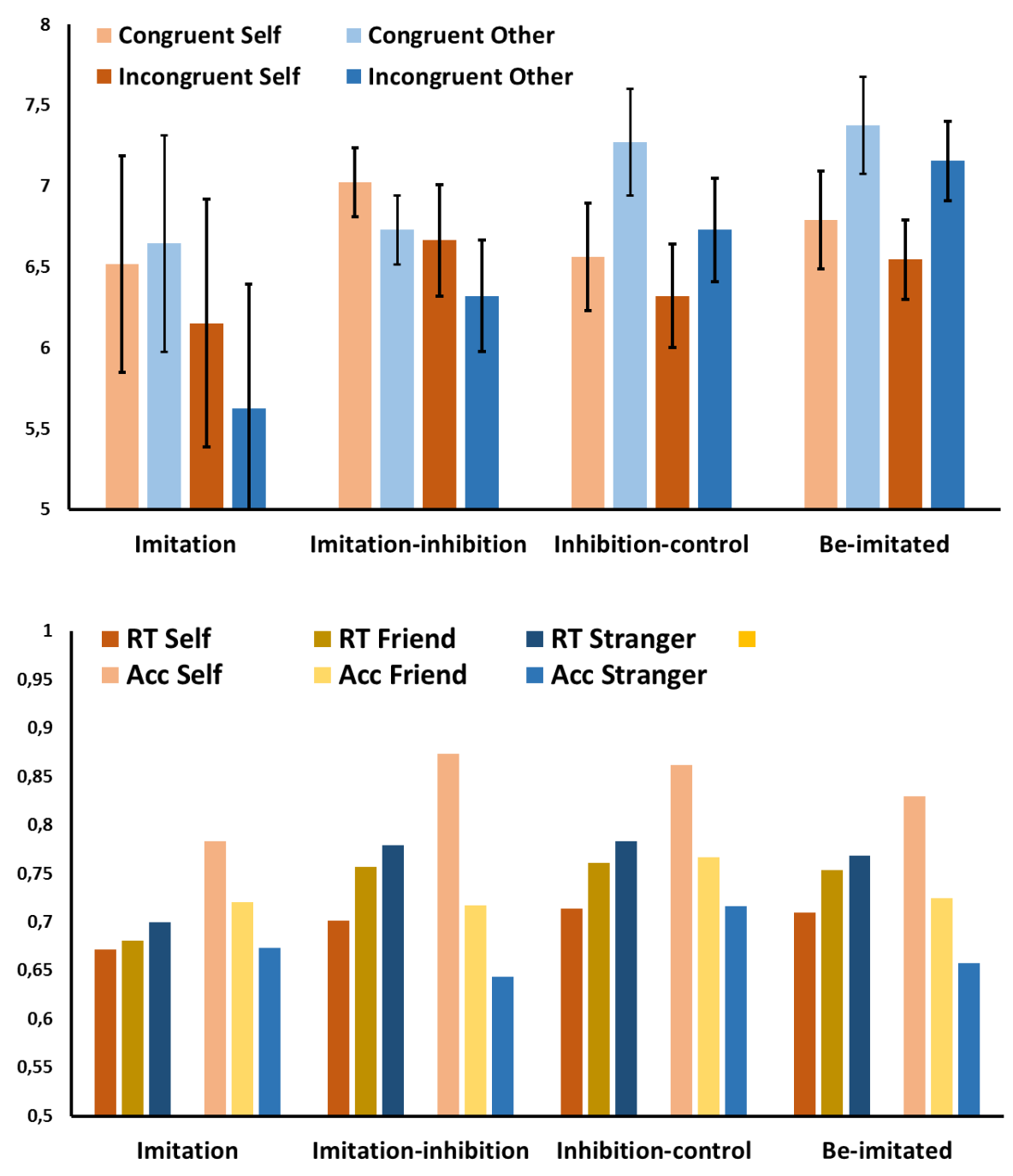

Figure 1. Impact of socio-cognitive training types on empathic and shape matching performance in experiment 1. Upper panel: Rating intensities when judging the self- and other person's emotional state when emotions are congruent and incongruent. Errors bars indicate pairwise within-subject 95\% confidence interval. Lower panel: Reaction times (ms divided by 1000 for illustrative purposes) and accuracy rates to verify matching shape-label parings for shapes associated with the self, the best friend, and a stranger.

\subsubsection{Shape matching performance}

Rates of correct responses $(M=.666, S D=0.165)$ of 22 participants were at chance level (50\% chance getting the right response; cut-off rate of .56 for 95\% confidence that performance is above chance) and were thus excluded from analyses. 


\section{EMOTIONAL \& PERCEPTUAL SALIENCE}

Reaction times (RT): The repeated-measures ANOVA revealed a significant linear main effect of shapes, $F(1,60)=35.258, p<.001, \eta_{p}{ }^{2}=.370$, with RT for the self being fastest and RT for stranger being the slowest, and a non-significant shape by training interaction, $F(3,60)=1.257$, $p=.297, \eta_{p}{ }^{2}=.059$. Building upon the increased emotional self-salience in imitation-inhibition, we tested whether perceptual self-salience (i.e., performance advantage to the self shape over the two other shapes) in the imitation-inhibition group would differ from the other groups via a series of two-sided $t$-tests for independent samples. Although perceptual self-salience was numerically highest in the imitation-inhibition group $(M=67.027, S D=83.263)$, the difference was significant against the imitation group $(M=18.920, S D=46.483 ; p=.037)$ but not against the inhibitioncontrol $(M=57.575, S D=59.784 ; p=.692)$ and be-imitated groups $(M=51.139, S D=64.768 ; p$ $=.484)$. At last, there was a significant main effect of training groups, $F(3,60)=3.178, p=.030$, $\eta_{p}{ }^{2}=.137$, with faster RT in the imitation group.

Accuracy: The repeated-measures ANOVA revealed a significant linear main effect of shapes, $F(1,60)=47.962, p<.001, \eta_{p}^{2}=.444$, with the highest accuracy for the self and lowest for the stranger, and a non-significant shape by training interaction, $F(3,60)=1.174, p=.327, \eta_{p}^{2}=$ .055. As for the RT, we tested whether perceptual self-salience (i.e., performance advantage to the self shape over the two other shapes) in the imitation-inhibition group would differ from the other groups via a series of two-sided $t$-tests for independent samples. Although perceptual self-salience was numerically highest in the imitation-inhibition group $(M=.193, S D=.153)$, the difference was significant against the imitation group $(M=.086, S D=.095 ; p=.020)$ but not against the inhibition$\operatorname{control}(M=.121, S D=.135 ; p=.129)$ and be-imitated groups $(M=.138, S D=.127 ; p=.228)$. At last, there was no significant main effect of training groups, $F(3,60)=1.062, p=.372, \eta_{p}^{2}=.050$.

Overall, finding a perceptual self-salience on both RT and accuracy replicates the original study and indicates that the shape matching task was properly completed. However, there was no reliable statistical evidence for an impact of training on the shape matching performance. Exploratory results indicate however that the perceptual self-salience on both RT and accuracy is the highest in the imitation-inhibition, an observation already made for the emotional self-salience measured with the affective touch task.

\subsection{Intermediary discussion}

The data collected so far suggests that the socio-cognitive training has no reliable impact on empathic performance and shape matching performance. However, marginally significant and 


\section{EMOTIONAL \& PERCEPTUAL SALIENCE}

exploratory findings suggest self-salience is increased following imitation-inhibition training both in the emotional and the perceptual domains. If these results were confirmed it would question the initial finding of Santiesteban et al. (2012) according to which perspective-taking performance was improved following imitation-inhibition due to enhanced self-other distinction. Aiming to replicate these preliminary findings with a higher sample size (three times the minimum sample of 11 participants per condition) we ran a second experiment with the identical procedure except that the be-imitated condition was dropped since findings from this condition could not be compared with the study of Santiesteban et al. (2012).

\section{Experiment 2}

\subsection{Material and methods}

\subsubsection{Sample:}

As in experiment 1 only female participants were recruited. This time we decided to triple the required sample size, and recruited 111 healthy adults, resulting in a final sample of 109 participants $\left(M_{\mathrm{age}}=21.58 ; S D_{\mathrm{age}}=3.71\right)$ after exclusion of two dropouts. The sample was randomly distributed between 3 training groups: the imitation $(N=37)$, imitation-inhibition $(N=36)$, and control-inhibition $(N=36)$ as in Santiesteban et al. (2012). Inclusion criteria were an absence of past or current psychiatric diagnosis. Participants received a course credit with the psychology bachelor curriculum in return for their participations.

\subsubsection{Materials and Procedure:}

Procedure and material are identical to experiment 1 except for the absence of the beimitated training condition.

\subsection{Results}

\subsubsection{Group differences}

Group differences in age and scores of the IRI, cEQ, and PANAS were inspected across the 3 priming groups by performing one-way ANOVAs with the training group membership as between-subject factor. Age data and questionnaires data of 2 participants ( 1 for cEQ) was missing. The ANOVA revealed non-significant difference between training groups for age $(F(2,107)=$ $1.583, p=.210)$, IRI perspective-taking $(F(2,107)=0.427, p=.654)$, IRI fantasy $(F(2,107)=0.636$, $p=.531)$, IRI empathic concern $(F(2,107)=0.034, p=.967)$, IRI global score $(F(2,107)=0.364$, $p=.696), \operatorname{cEQ}(F(2,108)=0.248, p=.781)$, PANAS positive affect $(F(2,107)=1.013, p=.367)$, 


\section{EMOTIONAL \& PERCEPTUAL SALIENCE}

and PANAS global score $(F(2,107)=1.309, p=.274)$. The training groups significantly differed for IRI personal distress $(F(2,107)=3.403, p=.037)$ and PANAS negative affect $(F(2,107)=$ $3.439, p=.036)$. Post-hoc analyses show that personal distress in the imitation group $(M=19.865$, $S D=4.097)$ is lower than in imitation-inhibition $(M=21.943, S D=4.646, p=.034)$ and inhibitioncontrol groups $(M=22.111, S D=3.487, p=.021)$ whereas negative affect in the imitationinhibition group $(M=19.371, S D=10.778)$ is higher than in the imitation $(M=14.162, S D=8.358$, $p=.012)$ and inhibition-control groups $(M=15.750, S D=6.124, p=.079)$. Given these group differences, the results on empathic and shape matching performance are also presented with personal distress and negative affect as covariates.

\subsubsection{Empathic performance}

Inspection of rates of erroneous valence ratings $(M=.027, S D=0.049)$ showed that no participant performed at chance level (50\% chance getting the right valence) and thus none was excluded from analyses.

The repeated-measures ANOVA of the ratings revealed a significant main effect of congruency, $F(1,99)=26.570, p<.001, \eta_{p}{ }^{2}=.212$, with a lower median emotional intensity in the incongruent emotions condition, a significant main effect of perspective, $F(1,99)=6.756, p=.011$, $\eta_{p}{ }^{2}=.064$, a non-significant congruency by perspective interaction, $F(1,99)=0.530, p=.468, \eta_{p}^{2}$ $=.005$. These findings replicate previous studies (Bukowski et al., 2019; Silani et al., 2013).

Of particular interests were the interactions of the socio-cognitive training types with the aforementioned effects. We found a non-significant congruency by training interaction, $F(2,99)=$ 2.086, $p=.130, \eta_{p}{ }^{2}=.040$, a non-significant perspective by training interaction, $F(2,99)=0.793$, $p=.455, \eta_{p}{ }^{2}=.016$, and a significant congruency by perspective by training interaction, $F(2,99)=$ $3.130, p=.048, \eta_{p}^{2}=.059$.

We inspected the triple interaction by first testing whether we replicate the finding from experiment 1 that self-salience on congruent trials significantly differed between training groups. The ANOVA with the training group membership as a between-subject factor failed to reach significance, $F(2,101)=2.073, p=.131$, despite self-salience for congruent trials being highest in the imitation-inhibition, as in experiment 1). The triple interaction was further examined by inspecting the plot of the ratings across all experimental conditions (see upper panel of Figure 2), which shows that the driving group difference lies in the altercentric bias (difference between 


\section{EMOTIONAL \& PERCEPTUAL SALIENCE}

congruent self-perspective trials and incongruent self-perspective trials). Congruently, an ANOVA on the altercentric bias revealed a significant group differences, $F(2,101)=3.862, p=.024$, and post-hoc analyses showed a higher altercentric bias in the imitation-inhibition $(M=1.083, S D=$ 1.789) than in the imitation $(M=0.276, S D=1.027, p=.011)$ and inhibition-control groups $(M=$ $0.385, S D=0.898, p=.029)$.

The repeated measures ANOVA with the personal distress as covariate increased the statistical significance of the triple interaction $(p=.037)$, did not affect the other two interactions with training, but revealed a marginal perspective (i.e., with emotional self-salience) by personal distress interaction, $F(1,98)=3.832, p=.053, \eta_{p}^{2}=.038$. We examined this interaction via a Pearson correlational analysis that showed a small but significant positive interaction between emotional self-salience and personal distress, $r(102)=.219, p=.027$.

The repeated measures ANOVA with the negative affect as covariate slightly reduced the statistical significance of the triple interaction $(p=.069)$, did not affect the other two interactions with training, and did not interact with any effect.

Overall, we found a significant impact of training on the altercentric bias, an index of selfother distinction performance, revealing an increased bias (i.e., reduced self-other distinction performance) in the imitation-inhibition group. Finally, we found again a high emotional selfsalience in the imitation-inhibition condition but only for congruent emotions trials. 

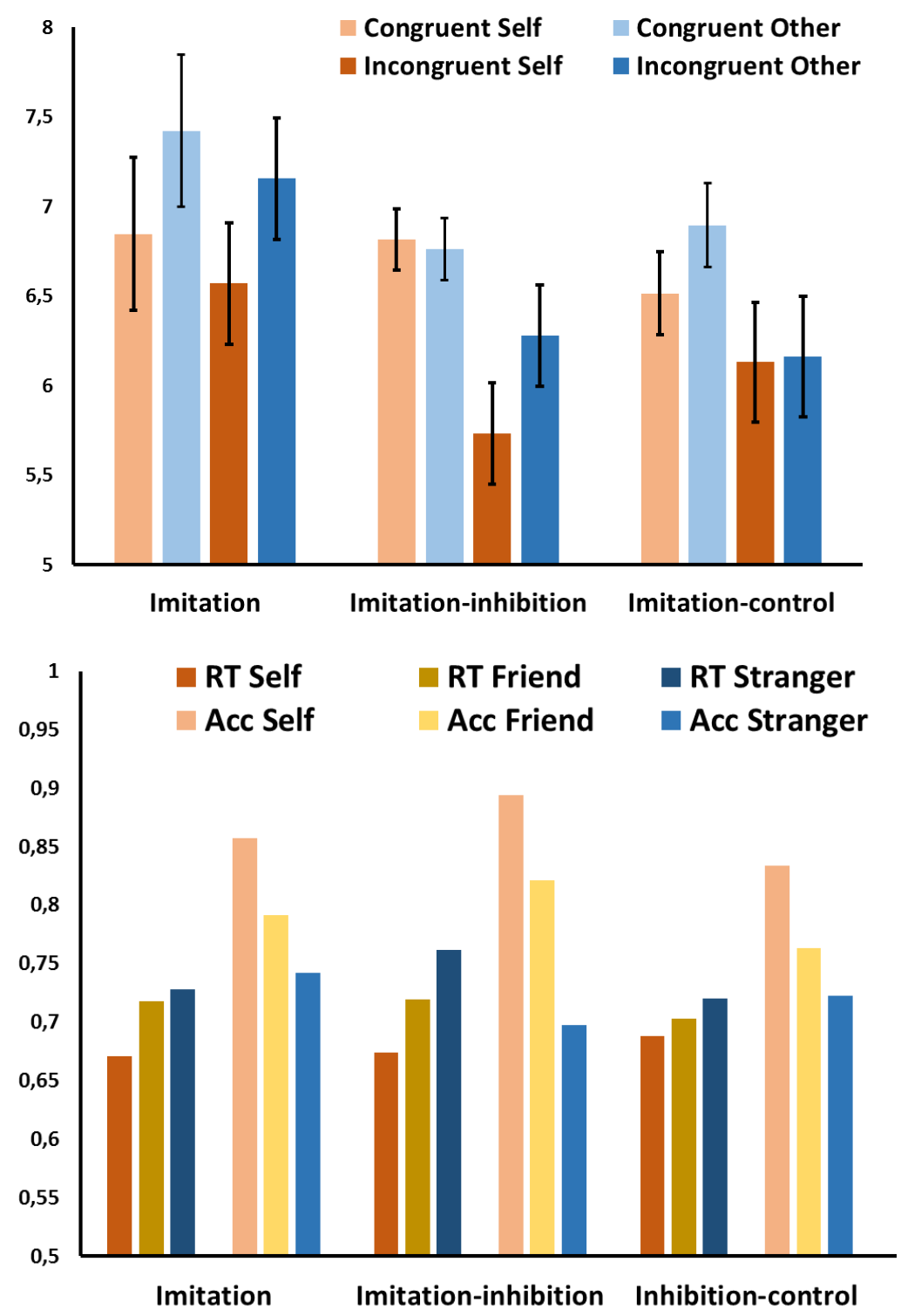

Figure 2. Impact of socio-cognitive training types on empathic and shape matching performance in experiment 2. Upper panel: Rating intensities when judging the self- and other person's emotional state when emotions are congruent and incongruent. Errors bars indicate pairwise within-subject 95\% confidence interval. Lower panel: Reaction times (ms divided by 1000 for illustrative purposes) and accuracy rates to verify matching shape-label parings for shapes associated with the self, the best friend, and a stranger.

\subsubsection{Shape matching performance}

Rates of correct responses $(M=.777, S D=0.011)$ of 6 participants were at chance level (50\% chance getting the right response; cut-off rate of .56 for $95 \%$ confidence that performance is above chance) and were thus excluded from analyses. 


\section{EMOTIONAL \& PERCEPTUAL SALIENCE}

Reaction times (RT): The repeated-measures ANOVA revealed a significant linear main effect of shapes, $F(1,94)=39.327, p<.001, \eta_{p}{ }^{2}=.295$, with RT for the self being fastest and RT for stranger being the slowest, and a marginally significant shape by training interaction, $F(2,94)$ $=2.850, p=.063, \eta_{p}{ }^{2}=.057$. Building upon the numerically high perceptual self-salience in imitation-inhibition found in experiment 1, we tested whether perceptual self-salience (i.e., performance advantage to the self shape over the two other shapes) in the imitation-inhibition group differed from the two other groups via two-sided $t$-tests for independent samples. Although perceptual self-salience was numerically highest in the imitation-inhibition group $(M=66.784, S D$ $=90.259)$, the difference was significant against the inhibition-control $(M=23.412, S D=59.813$; $p=.022)$ but not against the imitation group $(M=51.545, S D=68.751 ; p=.121)$. At last, there was no significant main effect of training groups, $F(2,94)=0.351, p=.705, \eta_{p}^{2}=.007$, with faster RT in the imitation group.

The repeated measures ANOVA with the personal distress as covariate slightly reduced the statistical significance of the shape by training interaction $(p=.080)$ and the personal distress by shape interaction was not significant $(p=.897)$. The repeated measures ANOVA with the negative affect as covariate slightly improved the statistical significance of the shape by training interaction $(p=.061)$ and the negative affect by shape interaction was not significant $(p=.441)$.

Accuracy: The repeated-measures ANOVA revealed a significant linear main effect of shapes, $F(1,94)=56.155, p<.001, \eta_{p}^{2}=.374$, with the highest accuracy for the self and lowest from the stranger, and a non-significant shape by training interaction, $F(2,94)=2.080, p=.131$, $\eta_{p}{ }^{2}=.042$. As for the RT, we tested whether perceptual self-salience (i.e., performance advantage to the self shape over the two other shapes) in the imitation-inhibition group would differ from the other groups via a series of two-sided t-tests for independent samples. Although perceptual selfsalience was numerically highest in the imitation-inhibition group $(M=.134, S D=.119)$, the difference was not significant against the imitation group $(M=.090, S D=.136 ; p=.167)$ and the inhibition-control $(M=.091, S D=.122 ; p=.182)$. At last, there was no significant main effect of training groups, $F(2,94)=0.972, p=.382, \eta_{p}^{2}=.020$.

The repeated measures ANOVA with the personal distress as covariate revealed a nonsignificant personal distress by shape interaction $(p=.407)$. The repeated measures ANOVA with the negative affect as covariate revealed a non-significant negative affect by shape interaction $(p=$ $.309)$. 


\section{EMOTIONAL \& PERCEPTUAL SALIENCE}

Overall, finding a perceptual self-salience on both RT and accuracy replicates the original

study (Sui, He, \& Humphreys, 2012) and indicates the shape matching task was properly completed. Although we found only a marginally significant impact of training on the shape matching performance, it replicated experiment 1 by showing that perceptual self-salience was the highest in imitation-inhibition on both RT and accuracy.

\section{Combined results}

Since procedures of experiment 1 and 2 were identical we re-examined the previously reported findings with two data sets combined except that data from the be-imitated of experiment 1 was excluded.

Sample: 178 female participants were allocated to either the imitation $(\mathrm{N}=62)$, imitationinhibition $(\mathrm{N}=62)$, or inhibition-control $(\mathrm{N}=54)$ training condition.

Age and questionnaires: No significant difference between the three training groups was found (all $p>$.123).

Empathic performance: The repeated-measures ANOVA of the ratings revealed a significant main effect of congruency, $F(1,168)=38.138, p<.001, \eta_{p}^{2}=.188$, a marginally significant main effect of perspective, $F(1,168)=3.456, p=.065, \eta_{p}{ }^{2}=.021$, a non-significant congruency by perspective interaction, $F(1,168)=0.296, p=.587, \eta_{p}^{2}=.002$, a non-significant congruency by training interaction, $F(2,168)=0.389, p=.679, \eta_{p}^{2}=.005$, indicating that self-other distinction was not impacted by training, a non-significant perspective by training interaction, $F(2,168)=0.864, p=.423, \eta_{p}^{2}=.010$, and a non-significant congruency by perspective by training interaction, $F(2,168)=2.004, p=.138, \eta_{p}{ }^{2}=.024$. However, a direct comparison of the selfsalience in congruent trials across the 3 training groups reached statistical significance on the ANOVA, $F(2,168)=4.064, p=.019$, and post-hoc analyses showed higher emotional self-salience following imitation-inhibition training $(M=0.157, S D=1.014)$ than the imitation training $(M=$ $0.428, S D=1.164 ; p=.020)$ and inhibition-control training $(M=-0.503, S D=1.299 ; p=.012)$.

Shape matching performance: The repeated-measures ANOVA on RT revealed a significant linear main effect of shapes, $F(1,144)=62.376, p<.001, \eta_{p}^{2}=.304$, and a significant shape by training interaction, $F(2,144)=3.097, p=.048, \eta_{p}^{2}=.042$, indicating more pronounced performance difference between the three shapes in the inhibition-imitation. Direct comparison of the perceptual self-salience across the 3 training groups reached marginal statistical significance 


\section{EMOTIONAL \& PERCEPTUAL SALIENCE}

on the ANOVA, $F(2,144)=2.766, p=.066$, and post-hoc analyses showed a higher perceptual self-salience following imitation-inhibition training $(M=66.869, S D=86.956)$ than the imitation training $(M=40.879, S D=63.779 ; p=.074)$ and inhibition-control training $(M=33.810, S D=$ $61.235 ; p=.028$; see Figure 3).

The repeated-measures ANOVA on accuracy revealed a significant linear main effect of shapes, $F(1,144)=91.913, p<.001, \eta_{p}{ }^{2}=.395$, and a significant shape by training interaction, $F(2,144)=3.803, p=.025, \eta_{p}{ }^{2}=.051$, indicating more pronounced performance difference between the three shapes in the inhibition-imitation. Direct comparison of the perceptual self-salience across the 3 training groups reached statistical significance on the ANOVA, $F(2,144)=3.634, p=.029$, and post-hoc analyses showed a higher perceptual self-salience following imitation-inhibition training $(M=.155, S D=.133)$ than the imitation training $(M=.089, S D=.123 ; p=.012)$ and inhibition-control training $(M=.100, S D=.125 ; p=.041$; see Figure 3$)$.

Overall, socio-cognitive training types have no tangible effect on self-other distinction performance but consistent effects on self-salience whereby both emotional and perceptual selfsalience is increased following imitation-inhibition training.

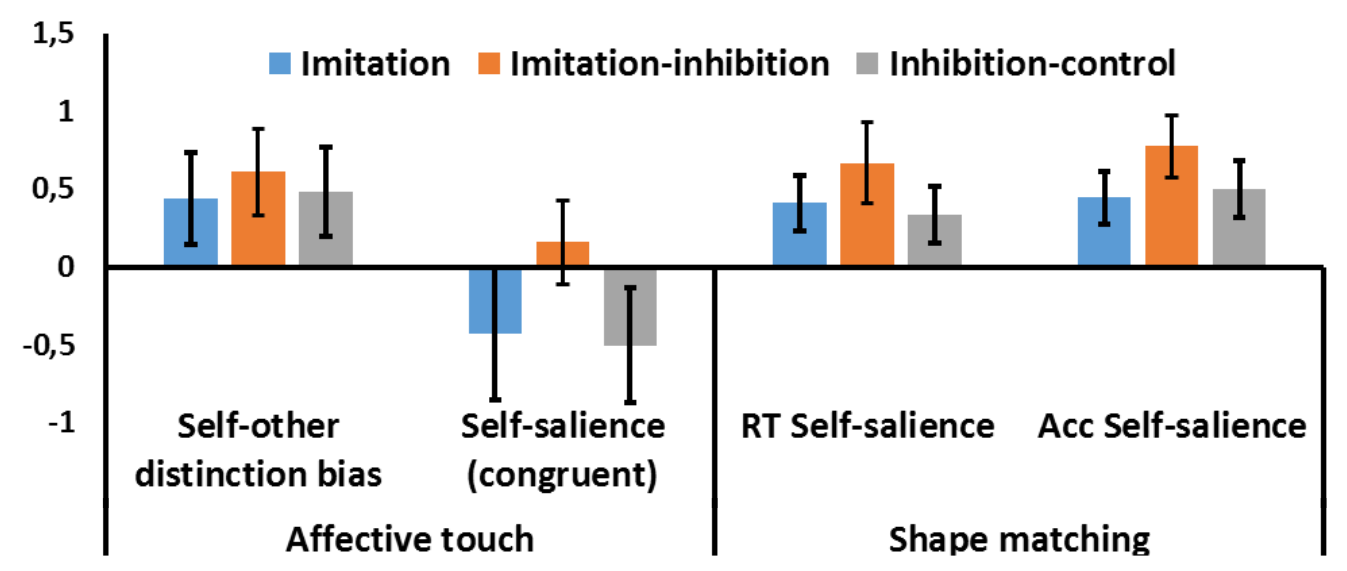

Figure 3. Impact of socio-cognitive training types on the self-other distinction bias and emotional self-salience measured with the affective touch paradigm and on the perceptual self-salience in terms of RT (ms divided 100 for illustrative purpose) and accuracy rate (multiplied by 5 for illustrative purpose). Errors bars indicate between-subject $95 \%$ confidence intervals. 


\section{EMOTIONAL \& PERCEPTUAL SALIENCE}

\section{Discussion}

Self-other distinction is crucial to prevent interferences from imitative tendencies and egocentric and altercentric bias in perspective taking and empathy, which raises the question of whether a multi-domain self-other distinction mechanism exists. Supporting evidence showed that training to inhibit imitation resulted in reduced imitation interferences and reduced egocentric bias in perspective taking (Santiesteban et al., 2012). However, the same training also improved empathy measures devoid of self-other distinction task-demands (de Guzman et al., 2015). This study examined the impact of the socio-cognitive training on an empathy measure of self-other distinction while testing the hypothesis that the training altered self-salience instead of self-other distinction. Comparison of the training types on empathic performance in experiment 1 revealed that self-other distinction was not enhanced by imitation-inhibition training, instead there was a trend for increased emotional self-salience. Moreover, perceptual self-salience was also numerically highest in imitation-inhibition training. Given the weak statistical reliability of the findings, a second experiment was conducted with a larger sample size. Experiment 2 replicated the absence of training effect on self-other distinction and the numerical superiority of emotional and perceptual self-salience in the inhibition-imitation training but still without reaching statistical significance. In addition, a significantly higher altercentric bias was found in the imitationinhibition. At last, combining the data from experiment 1 and 2 revealed statistically significant increases of emotional and perceptual salience following imitation-inhibition training while confirming the absence of training effect on self-other distinction.

The first hypothesis the present study tested was whether self-other distinction performance in empathy would be enhanced following imitation-inhibition training. Our results indicated that it was not the case; three potential explanations are considered respectively. The first potential explanation is that an underpowered sample size can cause false positive or false negative findings. However, our experiments doubled, then tripled the required sample size, resulting in 54 participants per training group. Hence, considering that the original study had 13 participants per training group, it seems more likely that original study's findings are a false positive than ours being a false negative. The second potential explanation is that the training effects on self-other distinction in automatic imitation and perspective taking do not generalize to self-other distinction in empathy. However, we could not identify any reason why self-other distinction in automatic imitation would generalize to perspective taking but not to empathy. Instead we posit that our 


\section{EMOTIONAL \& PERCEPTUAL SALIENCE}

measure of self-other distinction in empathy shares more features with the other two domains than the number of features shared between automatic imitation and perspective taking: Imitation is considered as an ontogenic precursor of empathy (but not of perspective taking) (Iacoboni, 2009) while the affective touch and perspective-taking paradigms (but not automatic imitation) measure egocentric and altercentric biases through explicit evaluations of self and another person's mental states.. The third and most likely explanation is that imitation inhibition did not enhance self-other distinction but affected performance in a different way, which is discussed below.

The second hypothesis the present study tested was whether self-salience would be altered following imitation-inhibition training. Our results indicated that this was indeed the case, but our finding raises two questions. First, although increased self-salience following imitation-inhibition training could explain why automatic imitation interference was reduced, it is harder to explain how it reduced egocentric inference in the visual perspective taking task of Santiesteban et al. (2012). However, it could be argued that the Director task used does not have trials requiring to take the self-perspective, which prevents comparisons between self and other trials to compute a proper self-salience index. Instead, we can only infer that self-salience when visual perspectives are conflicting (i.e., experimental trials in Director task) was not reduced following imitationinhibition. Interestingly, we actually found that indeed imitation-inhibition training did not impact the global emotional self-salience but only self-salience calculated on congruent trials, that is when perspectives are not conflicting. Moreover, examination of the plot of the increased altercentric bias found in experiment 2 shows that this a priori incoherent finding is explained by the increased intensity of ratings for self-congruent trials (i.e., the higher the average rating for self-congruent, the higher the difference from the average rating in self-incongruent). Finally, finding that imitation-inhibition training affected performance in absence of conflicting perspectives also explains why imitation-inhibition training had effects on empathy measures when no perspective conflict was present. The second to address is why imitation-inhibition training altered specifically self-salience on congruent trials. One aspect to consider is that, for both perceptual self-salience and emotional self-salience on congruent trials, self-prioritization occurred most likely on an early processing stage or at least on less high-level processing stages than when perspectives are conflicting (requiring awareness and regulation of the self-other difference). Another aspect to consider is a potential strategy the imitation-inhibition provokes. For instance, it is plausible that participants adopted a strong top-down self-focus, possibly on early sensory cues, to facilitate 


\section{EMOTIONAL \& PERCEPTUAL SALIENCE}

performance at resisting imitative tendencies. If so, such self-focus mindset could have gated early processes and could be effective for congruent perspectives trials but would be less effective when attentional demands must be diverted to resolve the self-other differences (i.e., incongruent trials). These explanations are however speculative and remain to be tested.

\section{Conclusions}

Our actions and thoughts are deeply influenced by other people's actions and thoughts. While self-other distinction is currently under the spotlight to explain these influences, it hides the fact that self-salience is an equally likely predictor of automatic imitation interferences, egocentric biases, and altercentric biases. This study demonstrates a case where an effect on self-other distinction should be reconsidered as an effect on self-salience. More importantly, we demonstrated it is feasible and useful to consider both self-salience and self-other distinction as two complementary angles to explain the past, present, and future self-other distinction findings. 


\section{EMOTIONAL \& PERCEPTUAL SALIENCE}

\section{Acknowledgments:}

We thank Rosalie Dittrich, Anja Tritt, Irena Tetkovic, Johann Börner, Julia Braunstein, Christoph

Frühlinger, and Jessica.zanner for their help in collecting the data. H.B., G.S., and C.L. designed

the study. H.B., B.T., and M.B. collected and analysed the data. All authors contributed to the manuscript writing.

Funding: Parts of this study were supported by the MOVE-IN Louvain and Marie-Curie actions cofounding scheme to H.B.. 


\section{EMOTIONAL \& PERCEPTUAL SALIENCE}

\section{References}

Brass, M., Bekkering, H., \& Prinz, W. (2001). Movement observation affects movement execution in a simple response task. Acta Psychologica, 106(1-2), 3-22. https://doi.org/10.1016/S0001-6918(00)00024-X

Brass, M., Bekkering, H., Wohlschla, A., \& Prinz, W. (2000). Compatibility between Observed and Executed Finger Movements : Comparing Symbolic, Spatial , and Imitative Cues, 143, 124-143. https://doi.org/10.1006/brcg.2000.1225

Bukowski, H. (2014). What influences perspective taking? A dynamic and multidimensional approach. Retrieved from http://hdl.handle.net/2078.1/151995

Bukowski, H., Silani, G., Riva, F., Tomova, L., \& Lamm, C. (2016). Measuring Self-Other Sharing and Self-Other Distinction as Distinct Dimensions of Empathy. In Poster presented at the 3rd international conference of the European Society for Cognitive and Affective Neuroscience, Porto, PL. (Vol. 2, p. 15466).

Bukowski, H., Tik, M., Silani, G., Ruff, C., Windischberger, C., \& Lamm, C. (2019). When differences matter: rTMS/fMRI reveals how differences in dispositional empathy translate to distinct neural underpinnings of self-other distinction in empathy. PsyArXiv. https://doi.org/10.31234/osf.io/9ndac

Cunningham, S. J., Turk, D. J., Macdonald, L. M., Macrae, C. N., \& Neil Macrae, C. (2008). Yours or mine? Ownership and memory. Consciousness and Cognition, 17(1), 312-318. https://doi.org/10.1016/j.concog.2007.04.003

De Coster, L., Andres, M., \& Brass, M. (2014). Effects of being imitated on motor responses evoked by pain observation: exerting control determines action tendencies when perceiving pain in others. The Journal of Neuroscience: The Official Journal of the Society for Neuroscience, 34(20), 6952-6957. https://doi.org/10.1523/JNEUROSCI.5044-13.2014

de Guzman, M., Bird, G., Banissy, M. J., \& Catmur, C. (2015). Self-other control processes in social cognition: From imitation to empathy. Philosophical Transactions of the Royal Society B: Biological Sciences, 371(1686), 20150079. https://doi.org/http://dx.doi.org/10.1098/rstb.2015.0079

Iacoboni, M. (2009). Imitation, empathy, and mirror neurons. Annual Review of Psychology, 60, 653-670. https://doi.org/10.1146/annurev.psych.60.110707.163604

Riva, F., Triscoli, C., Lamm, C., Carnaghi, A., \& Silani, G. (2016). Emotional egocentricity bias across the life-span. Frontiers in Aging Neuroscience, 8(APR), 1-7. https://doi.org/10.3389/fnagi.2016.00074

Rogers, T. B., Kuiper, N. A., \& Kirker, W. S. (1977). Self-reference and the encoding of personal information. Journal of Personality and Social Psychology, 35(9), 677.

Samson, D., Apperly, I. A., Braithwaite, J. J., Andrews, B. J., Bodley Scott, S. E., \& Scott, S. E. B. (2010). Seeing it their way: Evidence for rapid and involuntary computation of what other people see. Journal of Experimental Psychology: Human Perception and Performance, 36(5), 1255-1266. https://doi.org/10.1037/a0018729 
Santiesteban, I., White, S., Cook, J., Gilbert, S. J., Heyes, C., \& Bird, G. (2012). Training social cognition: From imitation to Theory of Mind. Cognition, 122(2), 228-235. https://doi.org/10.1016/j.cognition.2011.11.004

Silani, G., Lamm, C., Ruff, C. C., \& Singer, T. (2013). Right Supramarginal Gyrus Is Crucial to Overcome Emotional Egocentricity Bias in Social Judgments. The Journal of Neuroscience : The Official Journal of the Society for Neuroscience, 33(39), 15466-15476. https://doi.org/10.1523/JNEUROSCI.1488-13.2013

Sui, J., He, X., \& Humphreys, G. W. (2012). Perceptual effects of social salience: evidence from self-prioritization effects on perceptual matching. Journal of Experimental Psychology. Human Perception and Performance, 38(5), 1105-1117. https://doi.org/10.1037/a0029792

Sui, J., Humphreys, G. W., \& He, X. (2012). Perceptual effects of social salience: evidence from self-prioritization effects on perceptual matching. Journal of Experimental Psychology. Human Perception and Performance, 38(5), 1105-1117. https://doi.org/10.1037/a0029792

Tomova, L., Von Dawans, B., Heinrichs, M., Silani, G., Lamm, C., Dawans, B. Von, ... Lamm, C. (2014). Is stress affecting our ability to tune into others? Evidence for gender differences in the effects of stress on self-other distinction. Psychoneuroendocrinology, 43(July), 95104. https://doi.org/10.1016/j.psyneuen.2014.02.006 